\title{
An evaluation method of reflectance spectra to be obtained by Hayabusa 2 Near-Infrared Spectrometer (NIRS3) based on laboratory measurements of carbonaceous chondrites
}

\author{
Moe Matsuoka ${ }^{1 *} \mathbb{0}$, Tomoki Nakamura' , Takahito Osawa² ${ }^{2}$ Takahiro Iwata ${ }^{3}$, Kohei Kitazato ${ }^{4}$, Masanao Abe ${ }^{3}$, \\ Yusuke Nakauchi ${ }^{4}$, Takehiko Arai ${ }^{5}$, Mutsumi Komatsu ${ }^{6}$, Takahiro Hiroi ${ }^{7}$, Naoya Imae ${ }^{8,9}$, Akira Yamaguchi ${ }^{8,9}$ \\ and Hideyasu Kojima ${ }^{8,9}$
}

\begin{abstract}
We conducted ground-based performance evaluation tests of the Near-Infrared Spectrometer (NIRS3) onboard Hayabusa2 spacecraft in November 2013 and from April to May 2014 and established a method for evaluating its measured reflectance spectra. Reflectance spectra of nine powdered carbonaceous chondrite samples were measured by both NIRS3 and a Fourier transform infrared (FT-IR) spectrometer. We have established two methods for correcting the NIRS3 data by comparing them with the corresponding FT-IR data because raw data obtained by NIRS3 underwent spectral distortion caused by systematic offsets in sensitivity of individual pixels. The corrected NIRS3 spectra of carbonaceous chondrite samples are comparable with their FT-IR spectra. The depth of each band component $D_{\lambda}$ is defined for each wavelength $\lambda(\mu \mathrm{m})$ to characterize the absorption bands in NIRS3 spectra. It is suggested that the relationship between the $D_{2.72} / D_{2.79}$ ratio and the $D_{2.76} / D_{2.90}$ ratio would be useful for estimating the degree of heating of the asteroid surface, if contributions of terrestrial adsorbed water on $D_{2.79}$ and $D_{2.90}$ are properly corrected. The degrees of heating and space weathering are also comprehensively evaluated by the relationship between $D_{2.90}$ and the $D_{2.76} / D_{2.90}$ ratio. Reflectance spectra of asteroid Ryugu, the target asteroid of Hayabusa2, to be recorded by the NIRS3 instrument are expected to reveal the characteristics of the surface materials by using the evaluation technique proposed in this paper. Such information will be used for choosing the touchdown points for sampling and also for investigating the distribution of the materials similar to the returned samples on Ryugu.
\end{abstract}

Keywords: Hayabusa2, Spectroscopy, NIRS3, Chondrites, Asteroids

\section{Introduction}

C-type asteroid 162173 Ryugu was selected as a target of Hayabusa2 mission of the Japan Aerospace Exploration Agency (JAXA) to study the origin and evolutional history of the solar system and the origin of organic matters available for life. The asteroid explorer Hayabusa2 was launched on December 3, 2014, and is scheduled to

\footnotetext{
*Correspondence: mmatsuoka@dc.tohoku.ac.jp

1 Tohoku University, Sendai, Miyagi 980-8578, Japan

Full list of author information is available at the end of the article
}

arrive at the target asteroid in June-July 2018. The sampler of Hayabusa2 is expected to collect rock samples from the surface of the asteroid during brief touchdowns. Hayabusa2 has a multiband imager called Optical Navigation Camera Telescope (ONC-T), Near-Infrared Spectrometer (NIRS3), and Thermal Infrared Imager (TIR) among other instruments which will observe the asteroid in detail at a $20 \mathrm{~km}$ altitude (Tsuda et al. 2013). The spectroscopic observations of the asteroid are important for studying the compositional and physical properties 
of the asteroid surface which will help in selecting the touchdown points for sampling and also clarifying the relationship between the C-type asteroids and hydrous carbonaceous chondrites. The parent bodies of carbonaceous chondrites are still unclear, but spectroscopic studies for carbonaceous chondrites revealed that their ultraviolet (UV)-visible (Vis)-near-infrared (NIR) spectra are similar to those of $\mathrm{C}$-type asteroids, showing that the C-type asteroids are possible candidates as the parent bodies of CM chondrites (e.g., Vilas and Gaffey 1989; Hiroi et al. 1993, 1996; Vilas et al. 1994; Burbine et al. 2002; Lantz et al. 2013; Takir et al. 2013). Ground-based observations of asteroid Ryugu indicated that its surface materials would possibly consist of unheated CM chondrite (Vilas 2008) based on an observation of the 0.7$\mu \mathrm{m}$ band, or heated carbonaceous chondrite because its Vis-NIR spectrum corresponds with the spectrum of Murchison CM2 chondrite heated at $550-900{ }^{\circ} \mathrm{C}$. Their spectral slopes around $0.7 \mu \mathrm{m}$ are very similar to each other (Sugita et al. 2013; Perna et al. 2017).

At the long wavelength end of NIR range, the 3- $\mu \mathrm{m}$ band can be utilized to detect the condition of hydrous materials of asteroid surface because a strong $\mathrm{O}-\mathrm{H}$ stretching vibration band appears at $2.78 \mu \mathrm{m}$ (Clark et al. 1990) and the characteristic broad absorption is observed on many C-type asteroids (Jones et al. 1990; Rivkin et al. 2003, 2006; Takir et al. 2013). Okamura et al. (2014) suggested that the $3-\mu \mathrm{m}$ band depth of dark asteroids reflects the serpentine abundance based on IR spectra observed by the Japanese infrared astronomical satellite AKARI compared with the spectra of carbonaceous chondrites. Takir and Emery (2012) used the 3- $\mu \mathrm{m}$ band shape and the center wavelength to divide 28 outer main-belt asteroids into four groups based on spectra obtained using the SpeX spectrograph/imager at the NASA Infrared Telescope Facility (IRTF). The $3-\mu \mathrm{m}$ band can also be used to classify the meteorite types and estimate the degree of heating (Miyamoto and Zolensky 1994; Nakamura et al. 2015). Osawa et al. (2005) also proposed a spectroscopic taxonomy for carbonaceous chondrites using infrared transmission spectra. The absorption band reflects the state and concentration of water and is usually composed of two peaks: a sharp peak centered at $\sim 2.8 \mu \mathrm{m}$ due to the presence of structural water and a broad peak at around $3.0 \mu \mathrm{m}$ due to the molecular water (Osawa et al. 2005). The 3- $\mu \mathrm{m}$ band of hydrous carbonaceous chondrites is mainly controlled by the presence of hydrated minerals such as serpentines, saponite, and tochilinite that are observed in primitive carbonaceous chondrites (Zolensky et al. 1993). The shape of the $3-\mu \mathrm{m}$ band of an asteroid is thought to depend on three main factors: the composition of hydrous minerals, heating temperature, and the degree of space weathering. It is known that the depth of the 3- $\mu \mathrm{m}$ band of hydrous carbonaceous chondrites drastically decreases by heating due to the dehydration and decomposition of phyllosilicates (Hiroi et al. 1996), indicating that the $3-\mu \mathrm{m}$ band depth can be applied as an index of the heating stage. Hiroi et al. (1996) reported that the 3- $\mu \mathrm{m}$ band of powdered Murchison becomes shallower and rounded as heating progresses, and the $0.7-\mu \mathrm{m}$ absorption, which arises from $\mathrm{Fe}^{3+}-\mathrm{Fe}^{2+}$ charge transfers in Fe-rich hydrous mineral, vanishes faster than the 3- $\mu \mathrm{m}$ absorption. On the other hand, space weathering may also significantly alter the NIR spectra of C-type asteroids. The result of a space weathering simulation using nanosecond pulse-laser irradiation reveals that both the 0.7 - and 3- $\mu \mathrm{m}$ absorption bands of Murchison meteorite significantly weaken in accordance with the intensity of the laser irradiation (Matsuoka et al. 2015). Recording the reflectance spectra of Ryugu using spectrometers onboard Hayabusa2 spacecraft could detect the effects of the three factors mentioned above: the abundance and composition of hydrous minerals, thermal alteration, and space weathering. NIRS3 instrument onboard Hayabusa2 spacecraft can detect the $3-\mu \mathrm{m}$ band and observe hydrous minerals on the Ryugu surface (Kitazato et al. 2015; Iwata et al. 2017). In this paper, we introduce data correction and analysis methods for obtaining the degree of hydration from the $3-\mu \mathrm{m}$ band of the reflectance spectra of carbonaceous chondrites measured by NIRS3 as its prelaunch test.

\section{Experimental}

Since the mechanism and performance of NIRS3 are reported in detail by Iwata et al. (2017), here we briefly describe its structure. NIRS3 is composed of two component units: the spectrometric unit (NIRS3-S) and the analog electric unit (NIRS3-AE), which are connected with a harness cable (NIRS3-HNS). A 128-channel indium arsenide (InAs) photodiode sensor is installed in the spectrometric unit and cooled at $193 \mathrm{~K}\left(-80{ }^{\circ} \mathrm{C}\right)$ using a passive radiator. The detectable wavelength range of the spectrometer is $1.8-3.2 \mu \mathrm{m}$, and the spectral resolution is $18 \mathrm{~nm}$. Integration time of each recording is selectable within the range of $10 \mu \mathrm{s}$ to $10 \mathrm{~ms}$, and optimal integration time is $2.0 \mathrm{~ms}$ in this work.

Samples of seven CM chondrites, one CI chondrite, and one CV3 chondrite were used in this study. The meteorite samples are classified into four classes according to the degree of heating defined by Nakamura (2005) based on the results of X-ray diffraction analyses as follows.

1. Heating stage (HS)-I: unheated $\left(<300{ }^{\circ} \mathrm{C}\right)$ carbonaceous chondrites. A hydrous phase is mainly composed of serpentine and tochilinite. Murchison CM2 and Murray CM2 are classified into this class. 
2. HS-II: moderately heated $\left(300-500{ }^{\circ} \mathrm{C}\right)$ carbonaceous chondrites. Serpentine is decomposed into an amorphous state. Dehydration is not complete. Yamato (Y-) 793321 CM2, Jbilet Winselwan CM2, Y 982086 CM2, and Y $980115 \mathrm{CI} 1$ are in this class.

3. HS-IV: strongly heated $\left(>750{ }^{\circ} \mathrm{C}\right)$ carbonaceous chondrites. Hydrous minerals are almost completely dehydrated, and secondary anhydrous minerals that formed at the expense of amorphous silicates are dominant. Belgica (B-) 7904 CM2 and Y-86720 CM2 are in this class.

4. Anhydrous: Carbonaceous chondrites consist of anhydrous minerals almost entirely exclusive of hydrous minerals. Allende CV3 is in this class.

Meteorite chips were powdered with a tungsten carbide mortar and a pestle, and passed through a $155-\mu \mathrm{m}$ sieve for all meteorite samples and a 77- $\mu \mathrm{m}$ sieve only for Murray, Y-793321, and B-7904. Each powder sample was placed in an alumite-coated dish of $30 \mathrm{~mm}$ in diameter and flattened by tapping on the side of the dish.

The reflectance spectral measurements of the carbonaceous chondrite samples were performed using the NIRS3 flight model at Meisei Electric Co., Ltd., Isesaki, Japan. Each carbonaceous chondrite sample was set in an acrylic fiber desiccator, which was evacuated to a pressure of $\sim 0.01 \mathrm{MPa}$ using a dry pump to minimize the adsorbed water from the sample surface. A blackbody furnace (cavity blackbody source; CS1250-100 manufactured by Electro Optical Industries), used as a temperature-stable light source, was heated at $1250{ }^{\circ} \mathrm{C}$. The geometry of the optical experiment is presented in Fig. 1. The optics comprise two gold mirrors, two $\mathrm{CaF}_{2}$ windows, and three apertures. The light beam was collimated by the three apertures, and the angles of incidence and emergence were set to $30^{\circ}$ and $0^{\circ}$, respectively. The transparent chassis was covered with a blackout curtain during the experiment to reduce the background signal. The size of measured area was about $30 \mathrm{~mm}$ in diameter on the surface of each sample, and the reference spectrum was obtained using Infragold diffuse reflectance standard manufactured by Labsphere Inc. The thermal radiation spectrum was also measured for each meteorite sample without incident light. The temperature of the sample surface was monitored by a type-K thermocouple during the measurement, and the increase of temperature by the incident light was estimated to be less than $2{ }^{\circ} \mathrm{C}$. The reflectance spectra of same samples were measured using Bruker VERTEX 70v FT-IR spectrometer at Tohoku University. The incidence and emergence angles were set to 30 and $0^{\circ}$, respectively. Details of the setting are described in Matsuoka et al. (2017).

\section{Data reduction}

The signal intensity for each pixel of the detector is expressed by a 16-bit digital number (DN), and the relationship between the DN $(S)$ and analog output voltage $(V)$ is expressed as follows.

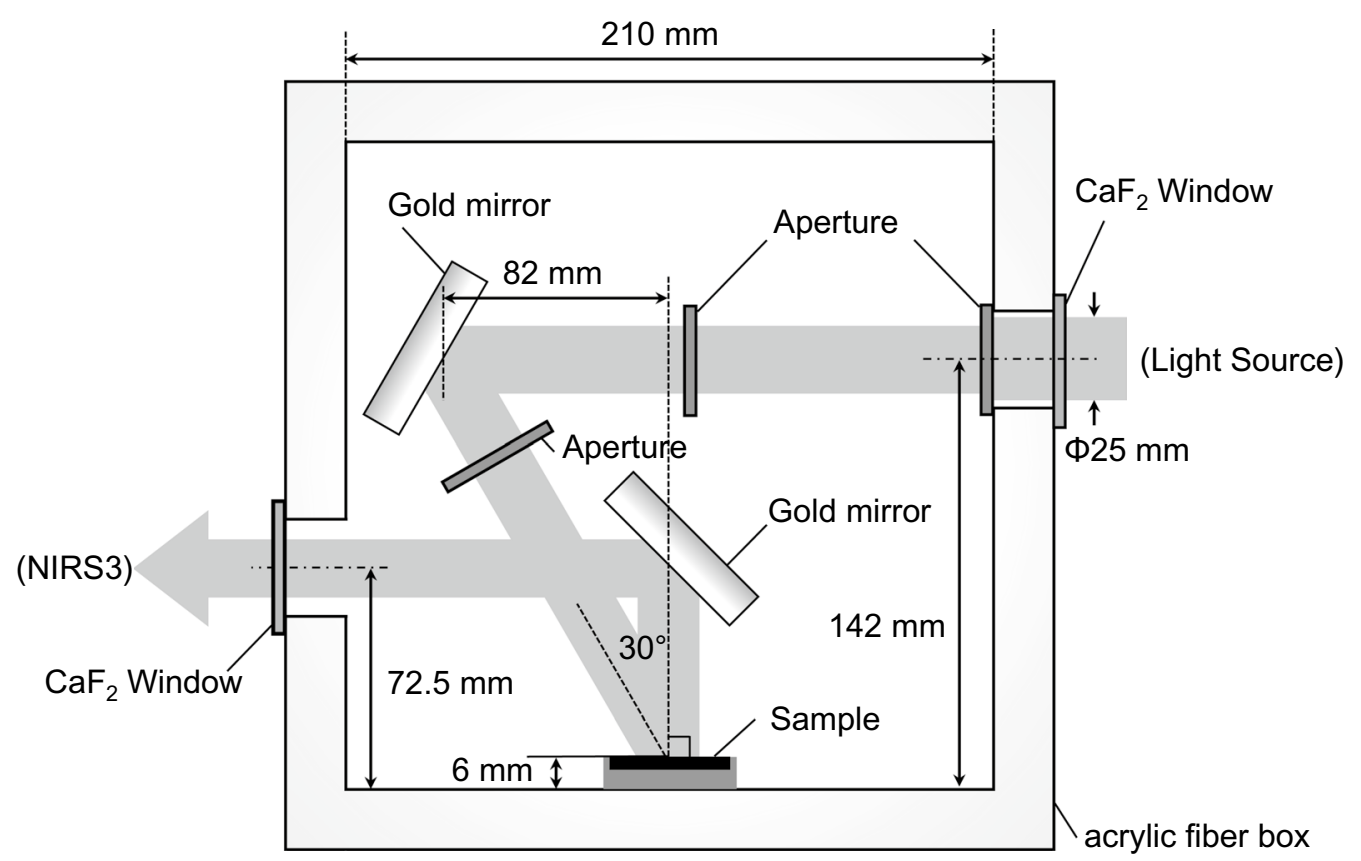

Fig. 1 Schematic diagram of the sample chamber for spectral measurements 


$$
S=\left\{\left(1.5 \times 2^{16}\right) / 5 \times V=1.966 \times 10^{4} \times V\right\}
$$

The $S$ value for each pixel is converted into the reflectance $(R)$ value as follows:

$$
\begin{aligned}
R= & \left(S_{\text {meteorite }}-S_{\text {thermal rad of met }}\right) / \\
& \left(S_{\text {infragold }}-S_{\text {thermal rad of std }}\right)
\end{aligned}
$$

The subscripts represent meteorite, thermal radiation of meteorite, reference, and thermal radiation of reference, respectively. The channel number $(n)$ of a 128-channel linear image sensor can be converted to wavelength $(\lambda$ $\mathrm{nm})$ as follows:

$$
\lambda=1230.555+18.55825 n-0.00487084 n^{2}
$$

The conversion formula was derived from the measurements using a monochrometer.

\section{Data correction methods}

Because individual pixels of the sensor array have different sensitivity characteristics from one another and receive light of different wavelengths, it is not easy to obtain a correct reflectance spectrum even though using a spectral standard such as Infragold. Indeed, the raw data recorded by NIRS3 have considerable spectral distortion and the spectral error may be caused by systematic offsets of the individual pixels. The two methods of spectral correction have been established in this work, and reflectance spectra of meteorites recorded by NIRS3 have been corrected by those methods. Although the two methods are different, their basic strategies are the same in that correction coefficients are determined by comparing the NIRS3 and FT-IR spectra of the same meteorite sample.

\section{Correction method 1}

Spectral data recorded by NIRS3 were corrected by the following steps.

1. In the first step, the NIRS3 and FT-IR spectra of the same meteorite sample were fit by linearly converting the FT-IR spectrum. When the absorption band at around $2.9 \mu \mathrm{m}$ is intense, there is a strong correlation between NIRS3 and FT-IR reflectance values as plotted in Fig. 2b. Their linear regression line (broken line) was calculated by the least squares method over the range of pixel numbers $40-100$, which corresponds to the range of $1.96-3.03 \mu \mathrm{m}$ in wavelength. The inclination and $y$-intercept of the regression line were used as the conversion parameters for fitting the FT-IR spectrum to the NIRS3 spectrum in Fig. 2a.

2. The difference in the signal intensity for each pixel between the NIRS3 and the fitted FT-IR spectra was computed. It is empirically known that the differences in the signal intensity are not due to statistical errors but to systematic gaps peculiar to each pixel, and therefore they are called systematic offsets in this paper. In order to obtain the systematic offsets, the signal differences were calculated for all samples measured in this work. The intensity of systematic offset for a pixel (pixel number is $\mathrm{n}$ ) was calculated by the formula.

$$
\begin{aligned}
I_{\text {offset }}(n)= & R_{\mathrm{FT}-\mathrm{IR}(\mathrm{fit})}(n) \times I_{\mathrm{NIRS} 3(\text { infragold })}(n) \\
& -I_{\mathrm{NIRS} 3(\text { meteorite) }}(n)
\end{aligned}
$$
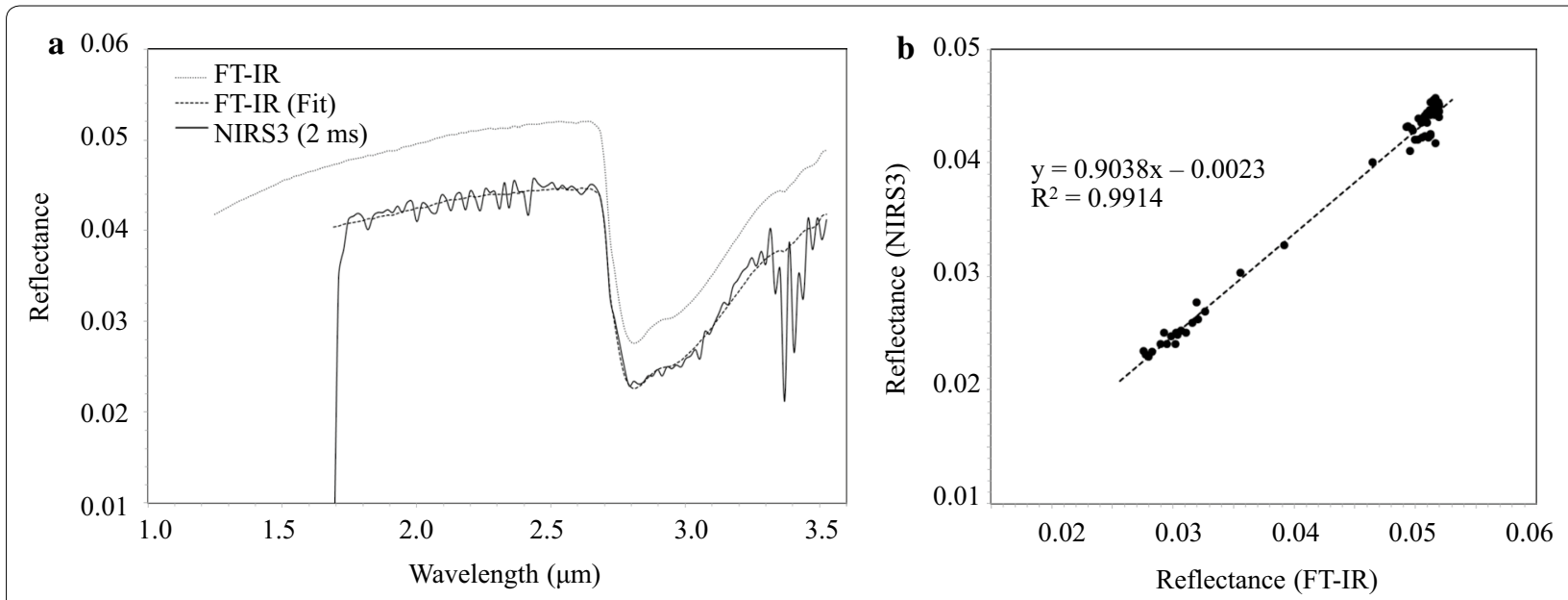

Fig. 2 Fitting process of FT-IR and NIRS3 spectra of Murray meteorite sample. a NIRS3, FT-IR, and fitted FT-IR spectra of Murray. a Relationship between the reflectance values by FT-IR and NIRS3 over the range of $1.96-3.03 \mu \mathrm{m}$ in wavelength. Integration time of the NIRS3 spectrum is $2.0 \mathrm{~ms}$. The FT-IR spectrum is fitted to NIRS3 spectrum using the correlation line plotted as a broken line in $\mathbf{b}$ 
Denoted as $I$ and $R$ are signal intensity and reflectance, respectively.

3. The systematic offsets are expressed as a linear function of signal intensity for each pixel (Fig. 3). NIRS3 spectra were corrected using these empirical laws of offsets and became significantly smoother after the elimination of the systematic offsets.

4. In the last step, reflectance values were corrected. The inclinations of the linear functions calculated in step (1) are roughly correlated with signal intensity, particularly the intensity of pixel number 29 (1.76 $\mu \mathrm{m}$ in wavelength) (Fig. 4a). The relationship between the inclinations and the signal intensity of the pixel is modeled by an exponential function. The $y$-intercepts of the linear functions are closely correlated with the inclinations; and its linear correlation coefficient is 0.902 (Fig. 4b). The NIRS3 spectra were thus corrected using the inclinations calculated from the exponential function and y-intercepts derived from the inclinations. The correction process of this method is summarized in Fig. 5.

\section{Correction method 2}

In the second correction method, reflectance spectra recorded by NIRS3 are corrected based on the FT-IR spectrum of the Allende sample. Since Allende meteorite has relatively low reflectivity and its reflectance spectrum has only a weak $\mathrm{O}-\mathrm{H}$ band (Fig. 6a), this meteorite may be appropriate as the spectral standard for correcting spectra of other carbonaceous chondrites. Because carbonaceous chondrites have low reflectivity, minerals with high reflectivity, for example serpentine, cannot be used as the spectral standard. NIRS3 spectra for a meteorite sample would be corrected by Eq. (5).

$$
\begin{aligned}
R(n) & =\frac{R_{\mathrm{FT}-\mathrm{IR}(\text { Allende) }}(n)}{R_{\mathrm{NIRS} 3 \text { (Allende) }}(n)} \times R_{\mathrm{NIRS} 3}(n) \\
& =S(n) \cdot R_{\mathrm{NIRS} 3}(n)
\end{aligned}
$$

NIRS3 reflectance $R_{\text {NIRS3 }}(n)$ of a meteorite sample is corrected to $R(n)$ using FT-IR reflectance $R_{\mathrm{FT}-\mathrm{IR} \text { (Allende) }}(n)$ and NIRS3 reflectance $R_{\text {NIRS3(Allende) }}(n)$ of the Allende sample, where $\mathrm{n}$ is a pixel number. $S(n)$ is a correction coefficient determined by the ratio of the reflectance of Allende meteorite measured by FT-IR and NIRS3 instruments (Fig. 6b). The spectra of all other meteorite samples can be corrected using the correction coefficient (e.g., Fig. 6c).

\section{Results and discussion}

Five diagrams depicted in Fig. 7 are reflectance spectra of nine carbonaceous chondrites recorded by FT-IR spectrometer (a) and NIRS3 (b, c, and d). Note that the spectra presented in Fig. $7 \mathrm{a}$ are converted spectra whose resolutions are reduced from 5 to $18 \mathrm{~nm}$ that corresponds to that of NIRS3. Both of those two correction methods can effectively correct the systematic offsets of NIRS3, and the corrected spectra are comparable with the FT-IR spectra. All carbonaceous chondrite samples other than Allende have clear 3- $\mu \mathrm{m}$ band, and the shapes of the absorption band distinctly reflect the state of the hydrous minerals in each meteorite. The $3-\mu \mathrm{m}$ absorption band can be attributed to the hydroxyl of $\mathrm{SiOH}$ in phyllosilicates, and the terrestrial adsorbed and rehydrated molecular water (e.g., Yamashita et al. 2015).
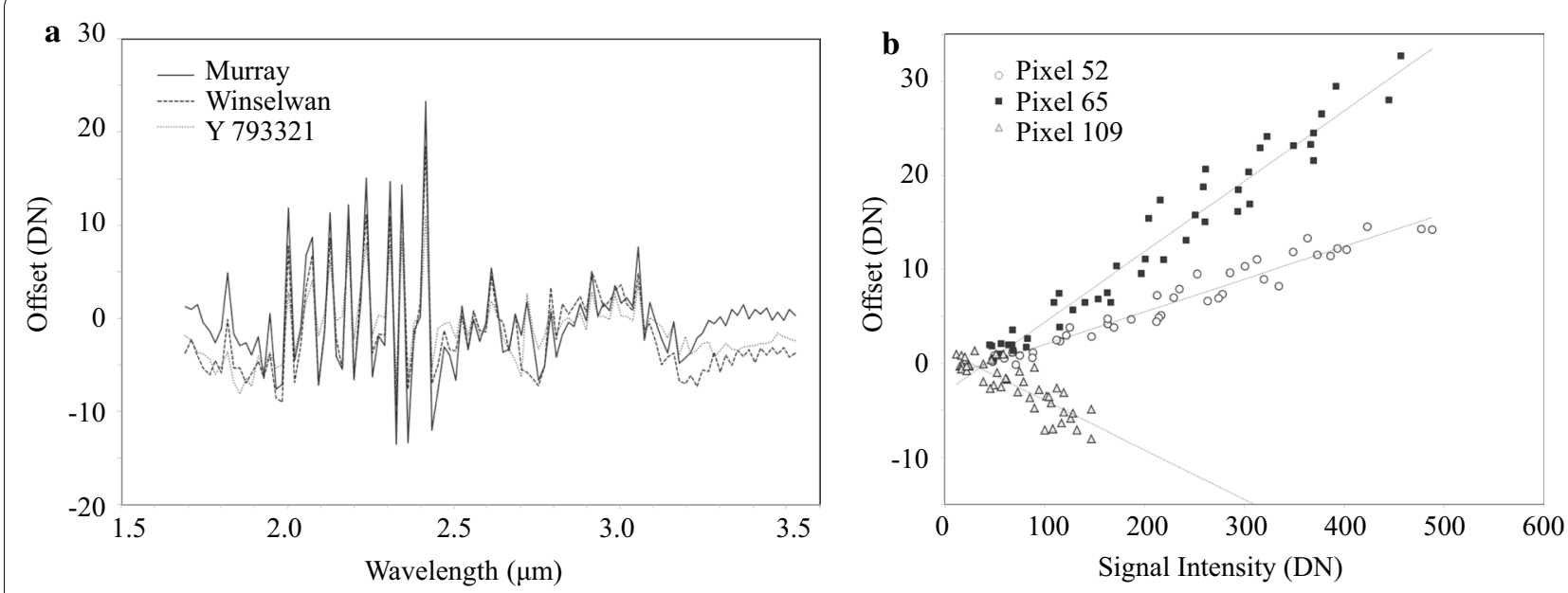

Fig. 3 Systematic offset values calculated from Eq. (4). a Offset value spectra over all the pixels for Murray, Jbilet Winselwan, and Y-793321 meteorite samples. b Relationship between the signal intensity and the offset value for three select pixels, using data for all the meteorite samples except Allende 

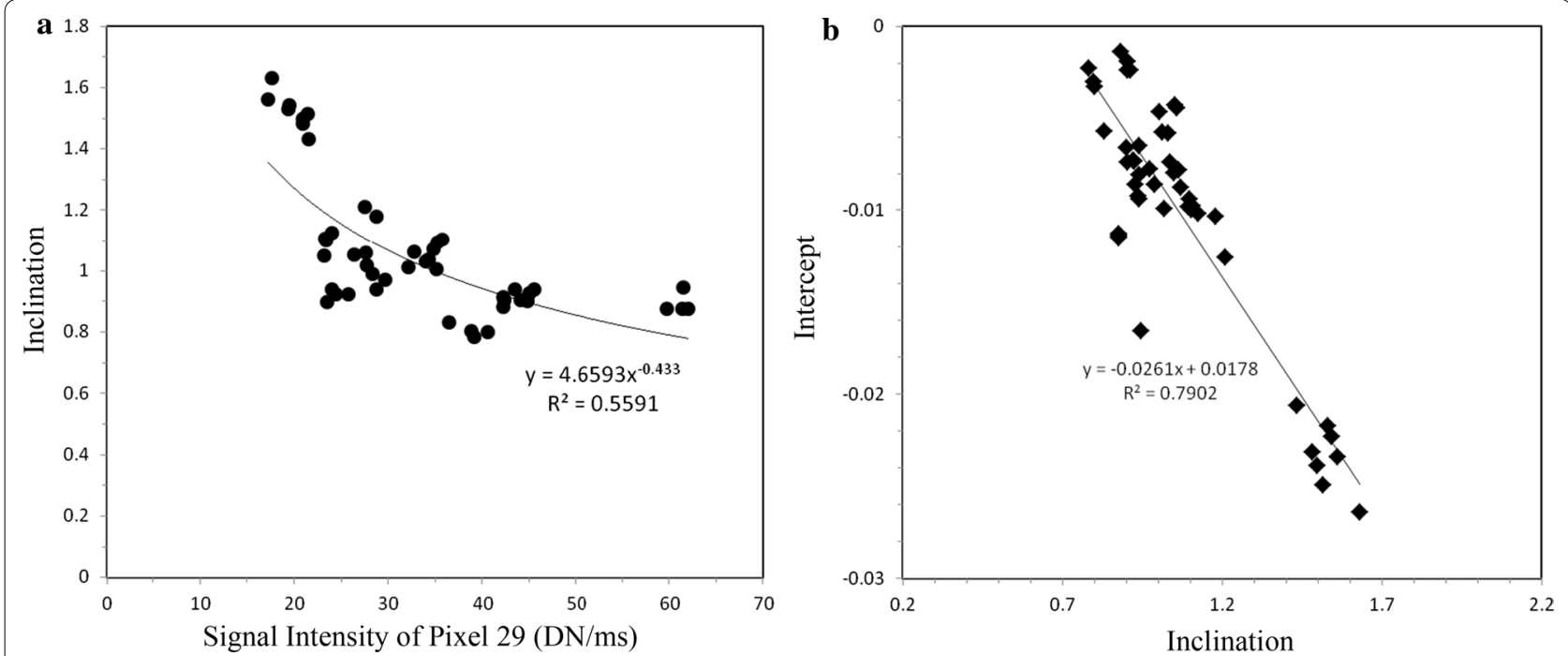

Fig. 4 Correlations among the signal intensity of pixel number 29, and the inclination and y-intercept values obtained by the linear regression analyses of NIRS3 and FT-IR reflectance data (e.g., Fig. 2b). The signal intensity is normalized by integration time. All meteorite data recorded by NIRS3 are used in $\mathbf{a}$, but the data of Allende are excluded in $\mathbf{b}$

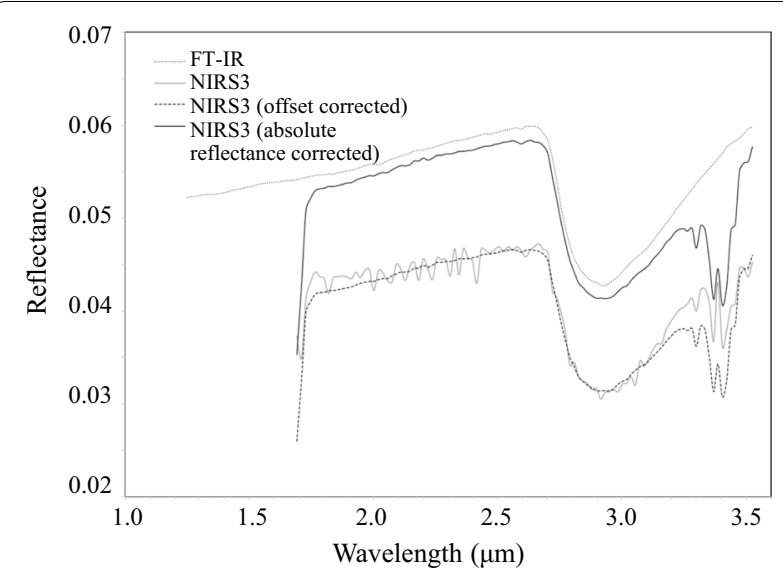

Fig. 5 Data correction process of reflectance spectrum of Y-86720 meteorite. NIRS3 reflectance data were corrected for the systematic offsets of pixel signals (broken line) and further corrected using the fitting parameters calculated from the signal intensity of pixel 29

(Fig. 3). The corrected spectrum (thick solid line) is very similar to the FT-IR spectrum (thin solid line)

The depth and shape of the 3- $\mu \mathrm{m}$ bands are correlated with the heating stage because of dehydration by heating. Samples of Murchison and Murray meteorite, which are classified into HS-I, have deep 3- $\mu \mathrm{m}$ band, showing only weak heating on the parent body. The HS-I chondrites have an asymmetric 3- $\mu \mathrm{m}$ band, which is composed of two well-defined peaks: One is at $2.79 \mu \mathrm{m}$, and the other is at $2.95 \mu \mathrm{m}$, indicating the presence of structural water and molecular water, respectively. On the other hand, the depths of the 3- $\mu \mathrm{m}$ band of HS-II chondrites (Y-793321,
Jbilet Winselwan, Y 982086, and Y 980115) are smaller than those of HS-I, and the peak centered at $2.79 \mu \mathrm{m}$ is weak except for Jbilet Winselwan. Only Jbilet Winselwan has a spike at $2.72 \mu \mathrm{m}$, reflecting the presence of structural hydroxyl group of clay minerals. Of the two HS-IV chondrites, there is a large difference between B-7904 and Y-86720; the $3-\mu \mathrm{m}$ band of B-7904 is very shallow, but the depth of the band of Y-86720 is comparable with that of Y 980115, which is classified into HS-II. The exceptional large absorption of Y-86720 is likely due to terrestrial weathering; Y-86720 could have reacted with Antarctic ice during the long-term storage under the ice sheet (Miyamoto 1992). An anhydrous chondrite, Allende meteorite, has considerably weak $3-\mu \mathrm{m}$ band, indicating the lack of water.

In order to analyze the absorption band more quantitatively, we performed modified Gaussian model (MGM) fitting calculations (Sunshine et al. 1990) for the $3-\mu \mathrm{m}$ band of FT-IR spectra (the range of wavelength is from 2.50 to $4.00 \mu \mathrm{m}$ ) using five modified Gaussian bands at $2.72,2.75,2.80,2.90$, and $3.22 \mu \mathrm{m}$. The 2.72 and $2.75 \mu \mathrm{m}$ bands are due to the stretching of the structural water in hydrous minerals, and 2.80, 2.90, and $3.22 \mu \mathrm{m}$ bands are due to the vibration of hydrogen bonded water. The FT-IR spectra were well fitted by the five bands (e.g., Fig. 8). However, the same fitting method cannot be applied to NIRS3 spectra because their longest wavelength is $3.2 \mu \mathrm{m}$. Therefore, the depth $\left(D_{\lambda}\right)$ of each band at wavelength $\lambda \mu \mathrm{m}$ is simply defined as follows:

$$
D_{\lambda}=1-R_{\lambda} / R_{2.5}
$$



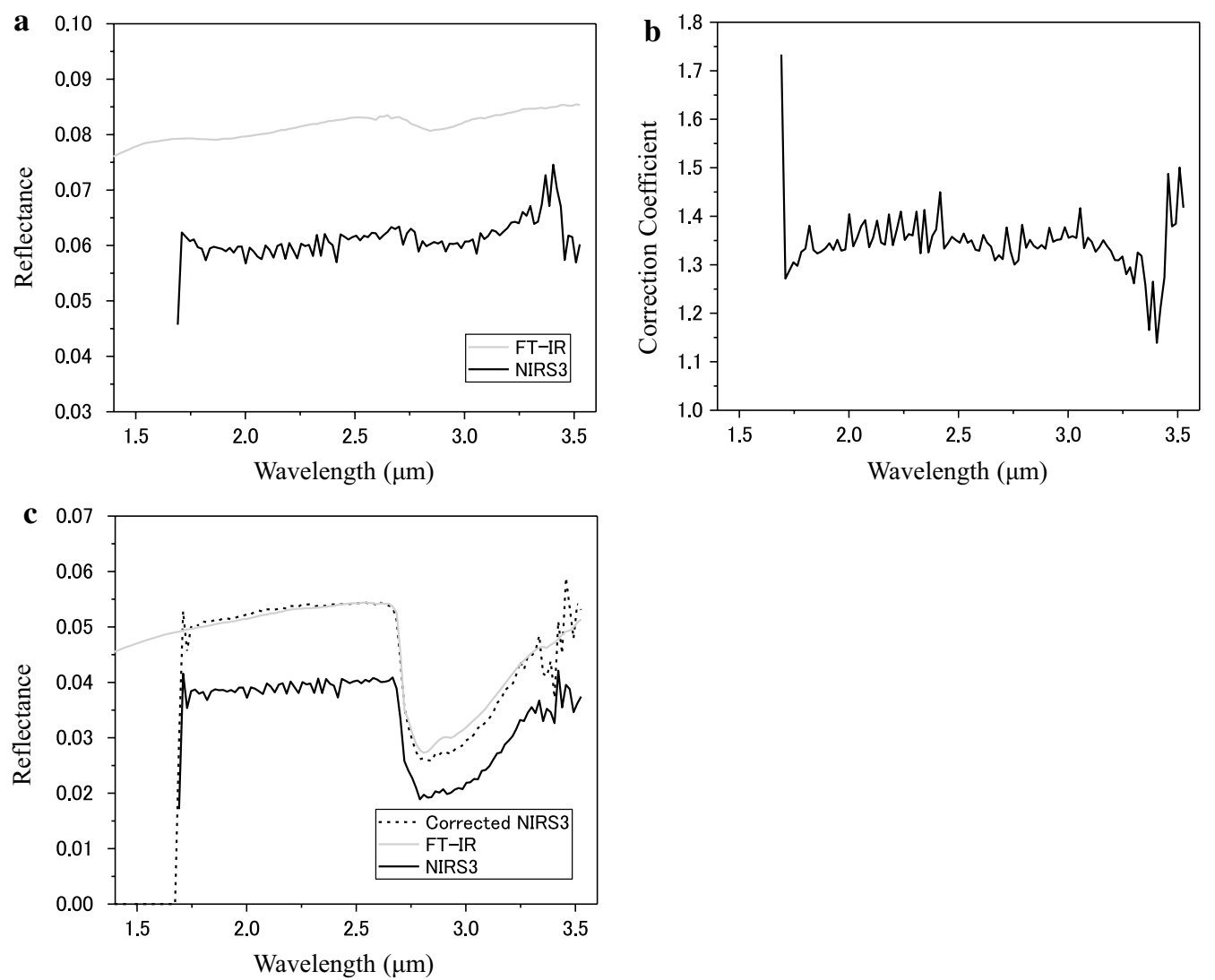

Fig. 6 Data correction process of reflectance spectrum of Murchison meteorite sample by the correction method 2. a NIRS3 and FT-IR spectra of Allende meteorite sample. $\mathbf{b}$ Correction coefficient based on the ratio reflectance values of Allende by FT-IR spectrometer and NIRS3 at each pixel. c FT-IR, NIRS3, and corrected NIRS3 spectra of Murchison sample

where $R_{\lambda}$ stands for reflectance at $\lambda(=2.72,2.76,2.79$, or $2.90 \mu \mathrm{m}$ ), the nearest values to the peak wavelengths of the modified Gaussian bands, and $R_{2.5}$ for reflectance at $2.5 \mu \mathrm{m}$ in wavelength as the reference point. Figure 9 shows the relationship between the $D_{2.72} / D_{2.79}$ ratio and the $D_{2.76} / D_{2.90}$ ratio of the eight carbonaceous chondrites samples. The $D_{2.72} / D_{2.79}$ ratio correlates well with the $D_{2.76} / D_{2.90}$ ratio, and it is likely that the distance from the origin to each data point roughly indicates their heating stage and weathering degree. Particularly, two HS-I chondrites (Murchison and Murray) and a HS-II chondrite (Jbilet Winselwan) are clustered above an arbitrary boundary line $\left(D_{2.76} / D_{2.90}=0.8\right)$ and are distinguishable from the other chondrites. Although the upper right cluster includes HS-I and HS-II meteorites, this diagram can be used to discriminate HS-I chondrites from other heated chondrites because chondrites similar to Jbilet Winselwan can be classified by its spectral shape. The figure thus can be used as a convenient method to evaluate the heating degree of the asteroid surface. However, this diagram cannot be used for discriminating between HS-II and HS-IV meteorite groups because this plot never reflects the depth of the 3- $\mu \mathrm{m}$ band, which is their major distinguishing factor.

The $D_{\lambda}$ values of NIRS3 spectra were directly compared with those of FT-IR spectra in order to establish another evaluation method. Figure 10 shows the relationships between the $D_{\lambda}$ values of FT-IR spectra and those of NIRS3 spectra corrected by method 1 or 2. The $D_{\lambda}$ values of NIRS3 spectra approximately correspond to those of FT-IR spectra, but the data points are slightly shifted from the solid line (slope $=1$ ), indicating the case where corrected NIRS3 data completely match with FT-IR data. The systematic difference in $D_{\lambda}$ values of NIRS3 data from FT-IR data could cause errors because the most NIRS3 data corrected by method 1 are systematically higher than the solid line in all diagrams and the NIRS3 data corrected by the method 2 are systematically lower than the method 1 data. However, these systematic errors can be corrected by modeling a linear correlation line between the FT-IR data and each of the NIRS3 data sets (Fig. 10d), and the difference between 


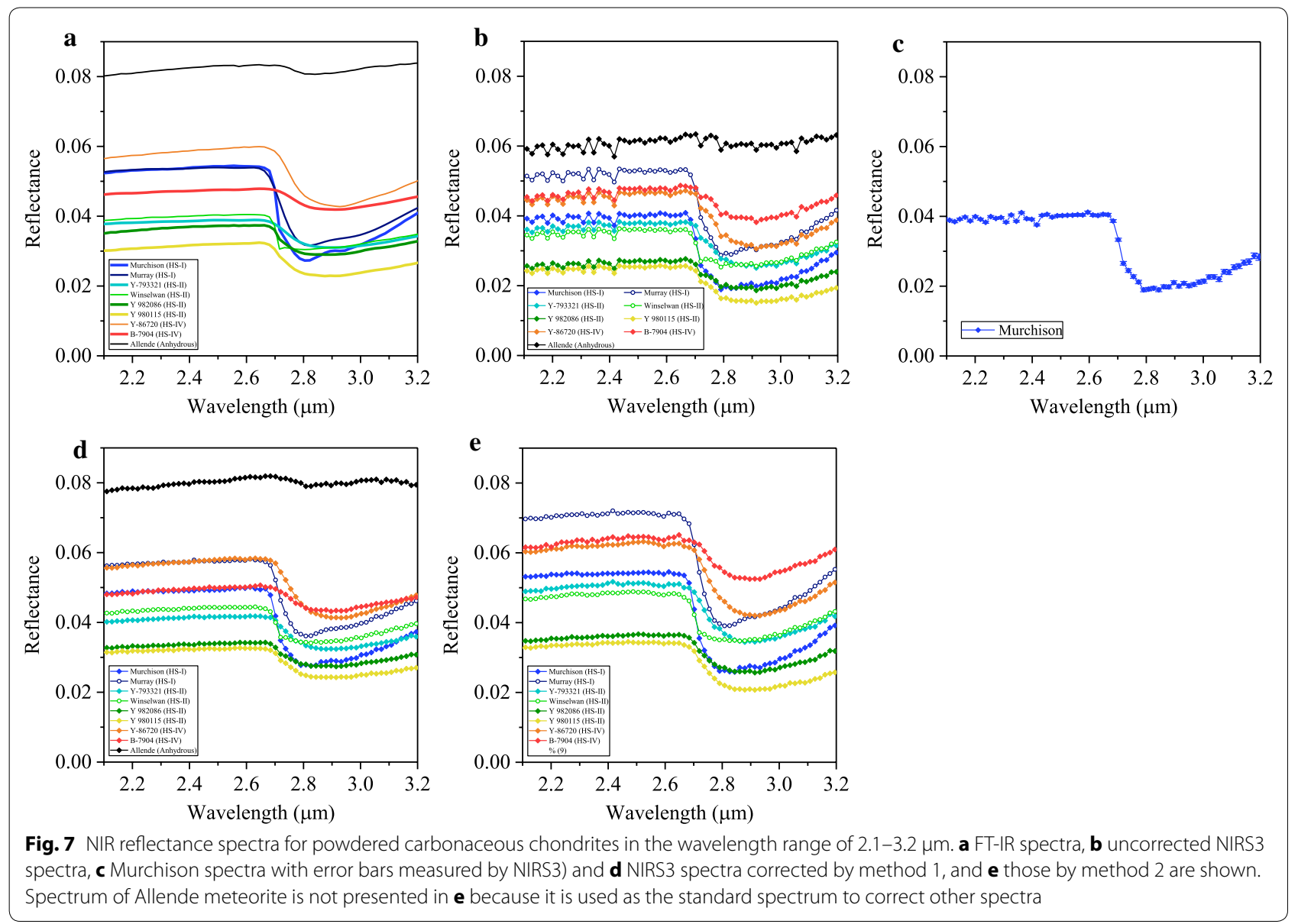

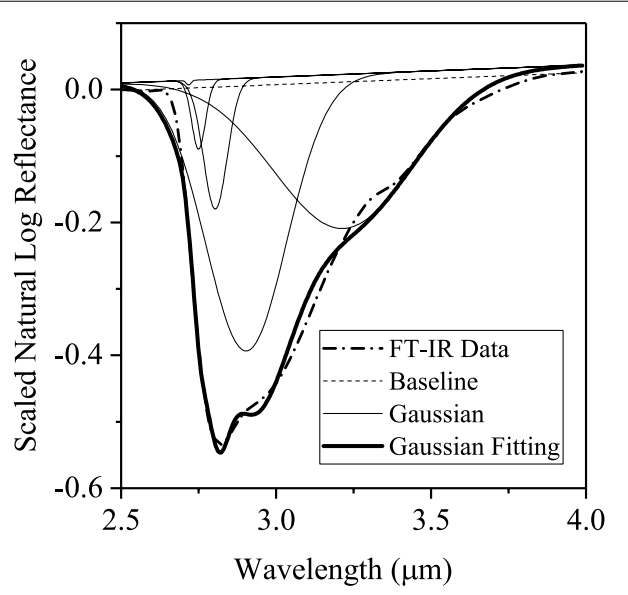

Fig. 8 MGM fitting of the $3-\mu m$ band of FT-IR spectrum of Murray sample those two correction methods can also be minimized. Figure 11 plots the $D_{2.90}$ values and the $D_{2.76} / D_{2.90}$ ratios after the $D_{2.90}$ values of NIRS3 data were corrected by the correlation lines (Fig. 10d). The $D_{2.90}$ values represent the depth of 3- $\mu \mathrm{m}$ band because $2.90 \mu \mathrm{m}$ is almost the center of the band. Note that $D_{2.90}$ values used in $D_{2.76} / D_{2.90}$ ratio on the vertical axis were not corrected. There are four clusters in this figure: HS-I aggregated in the upper right area, Jbilet Winselwan plotted in the left side of the HS-I area, B-7904 plotted in the lower left area, and other chondrites aggregated in the middle of the diagram. The data are not linearly distributed unlike in the case of Fig. 9, indicating that the distribution cannot be explained only by the effect of normal heating.

In order to compare the data with the results of heating and laser irradiation experiments for Murchison, we 


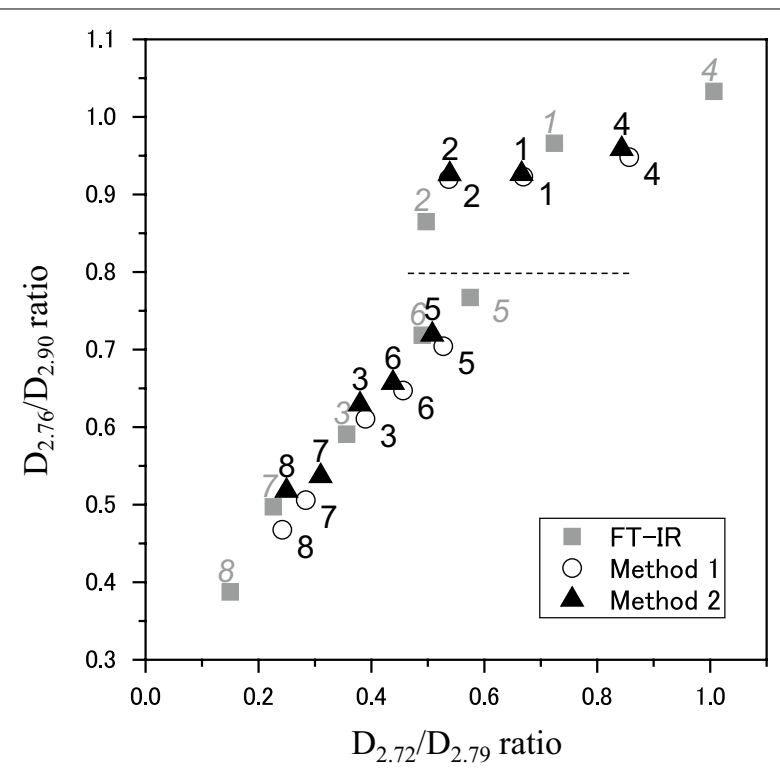

Fig. 9 Relationship between the $D_{272} / D_{279}$ ratio and the $D_{276} / D_{290}$ ratio. A broken line shows a gap at an arbitrarily $D_{2.76} / D_{2.90}$ value of 0.8 . The most hydrous chondrites $(1,2)$ and Winselwan $(4)$, which has a characteristic narrow peak at $2.72 \mu \mathrm{m}$, plot above the line. On the other hand, the other chondrites are clustered below the line. Labels: $1=$ Murchison, $2=$ Murray, $3=Y-793321,4=$ Jbilet Winselwan, $5=Y$ $982086,6=Y 980115,7=Y-86720$, and $8=B-7904$

made another diagram. Figure 12 shows the $D_{2.90}$ values and the $D_{2.76} / D_{2.90}$ ratios of NIRS3 data of the meteorite samples corrected by method 1, plotted with the corresponding data calculated from FT-IR spectra of Murchison samples heated in vacuum (up to $600{ }^{\circ} \mathrm{C}$, Hiroi et al. 1996) and irradiated by pulse laser (Matsuoka et al. 2015). The result of the heating experiment shows a characteristic shift in the figure; both $D_{2.90}$ and $D_{2.76} / D_{2.90}$ decrease proportionally from 400 to $600{ }^{\circ} \mathrm{C}$. The spectral transformation definitely relates to the mineralogical change, particularly progressive amorphization and dehydration of hydrous minerals, but its mechanism is not simple because the spectral transformation is controlled by the temperature characteristics of dehydration for each chemical state of water. However, the result of the spectral shift of the heated Murchison can be used to estimate the heating temperature of asteroid surface and other chondrites. Y 980115 is very close to the data of $400{ }^{\circ} \mathrm{C}$ heating, and B-7904 is between the data of 500 and $600{ }^{\circ} \mathrm{C}$. The results may reflect their heating temperature. Y-86720 is in the right side of the data of $500{ }^{\circ} \mathrm{C}$, which may be caused by the terrestrial weathering. Jbilet Winselwan and Y 982086 are plotted in the left side of the data of heated Murchison; especially Jbilet Winselwan is very far from the data of unheated Murchison. The large shift of $D_{2.90}$ may be due to the momentary impact or heating.

The pulse-laser irradiation experiment on Murchison $\mathrm{CM}$ chondrite was performed to simulate space weathering by micrometeorite bombardments (Matsuoka et al. 2015). The laser-irradiated Murchison samples with the output power ranging from 0 to $15 \mathrm{~mJ}$ clearly have lower $D_{2.90}$ values than the initial meteorite sample even though $D_{2.76} / D_{2.90}$ ratios are almost unchanged. The characteristic spectral change caused by the momentary heating (or impact) is quite different from the change of longterm heating. Although some mineralogical transformations are observed in the irradiated samples, for example amorphization, dehydration of the hydrous minerals, and deposition of FeS-rich amorphous silicate particles (Matsuoka et al. 2015), spectral shape is kept during laser irradiation in spite of a significant decrease of the depth of $3-\mu \mathrm{m}$ band. The mechanism of the spectral change is unclear, but it is important that the effect of momentary heating and long-term heating can be distinguished in the diagram because the vector of momentary heating is distinct from that of long-term heating. In order to analyze the observed data of Ryugu, it is necessary to take into account the effect of adsorbed and rehydrated water on $D_{2.79}$ and $D_{2.90}$ in this work, and also Figs. 11 and 12 should be modified without the contribution of adsorbed and rehydrated water in the future.

\section{Conclusions}

We propose here an evaluation procedure for the reflectance spectra of asteroid surface areas. In the first step, the shapes of obtained spectra are simply compared with those of powdered CM chondrite and are roughly classified into two classes such as typical CM and the other. Particularly Winselwan-type spectrum can be easily distinguished because it has a characteristic CI-like spectral shape. In the second step, heating degree is judged by using the relationship between the $D_{2.72} / D_{2.79}$ ratio and the $D_{2.76} / D_{2.90}$ ratio (Fig. 9). And in the last step, the degree of heating and space weathering are comprehensively evaluated by using the relationship between the $D_{2.90}$ value and the $D_{2.76} / D_{2.90}$ ratio (Fig. 11). We can conclude that NIRS3 has sufficient ability to evaluate the surface property of the CM-chondrite-like target asteroid because it has sensitivity for the wide range of wavelength up to $3.2 \mu \mathrm{m}$. In the previous observations, spectral heterogeneity of Ryugu was reported; Vilas (2008) suggested that a $0.7-\mu \mathrm{m}$ absorption band exists; on the other hand, 

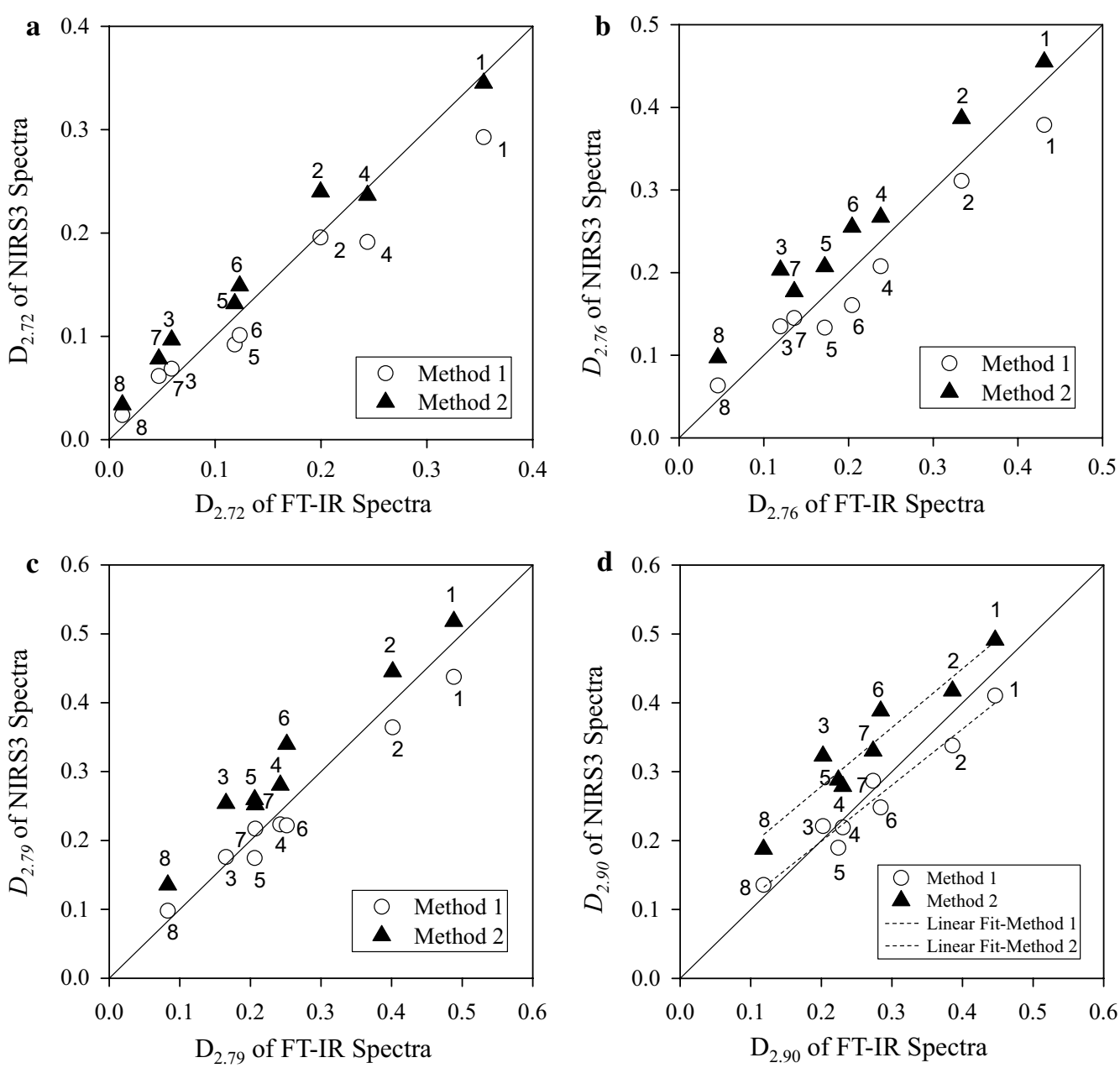

Fig. 10 Relationship of D values $\mathbf{a}$ at $2.72 \mu \mathrm{m}, \mathbf{b}$ at $2.76 \mu \mathrm{m}, \mathbf{c}$ at $2.79 \mu \mathrm{m}$, and $\mathbf{d}$ at $2.90 \mu \mathrm{m}$ between FT-IR spectra and NIRS3 spectra corrected by the method 1 (open circles) and the method 2 (filled triangles). Two broken lines in $\mathbf{d}$ show linear fits for the data. Labels: $1=$ Murchison, $2=$ Murray, $3=Y-793321,4=$ Jbilet Winselwan, $5=Y 982086,6=Y 980115,7=Y-86720$, and $8=B-7904$

spectra recorded by Bus and Binzel (2002), Lazzaro et al. (2013), Sugita et al. (2013), and Perna et al. (2017) showed no such absorption. The results may indicate that either the surface of Ryugu is extremely heterogeneous or a short-lived resurfacing event excavated hydrous minerals showing the $0.7-\mu \mathrm{m}$ absorption band. It is expected that reflectance spectra recorded by NIRS3 will decode the mystery using the evaluation technique established in this work. The obtained spectral information will also provide significant information for selecting Hayabusa2's touchdown points. Along with observations using other instruments on Hayabusa2, NIRS3 may reveal important 


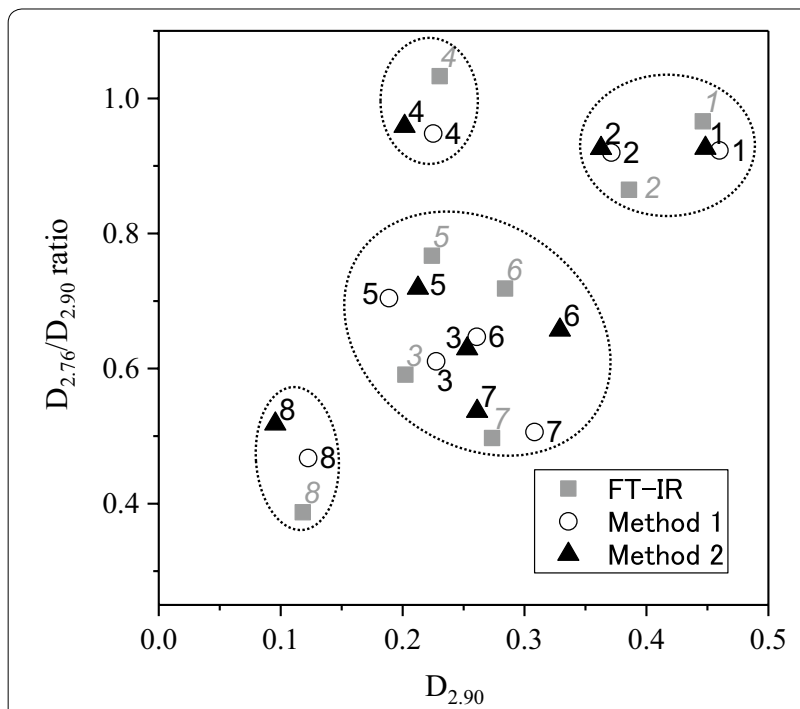

Fig. 11 Relationship between the $D_{2.90}$ values and the $D_{2.75} / D_{2.90}$ ratio. a The $D_{2.90}$ values of NIRS3 are corrected using the correction lines shown in Fig. 10d. Labels: $1=$ Murchison, $2=$ Murray, $3=\mathrm{Y}-793321,4=\mathrm{Jbilet}$ Winselwan, $5=\mathrm{Y} 982086,6=\mathrm{Y} 980115$ $7=Y-86720$, and $8=B-7904$. Surrounded by broken lines are four clusters: Highly hydrous chondrites (1 and 2), Jbilet Winselwan (4), moderate dehydrated chondrites $(3,5-7)$, and severely dehydrated chondrite (8)

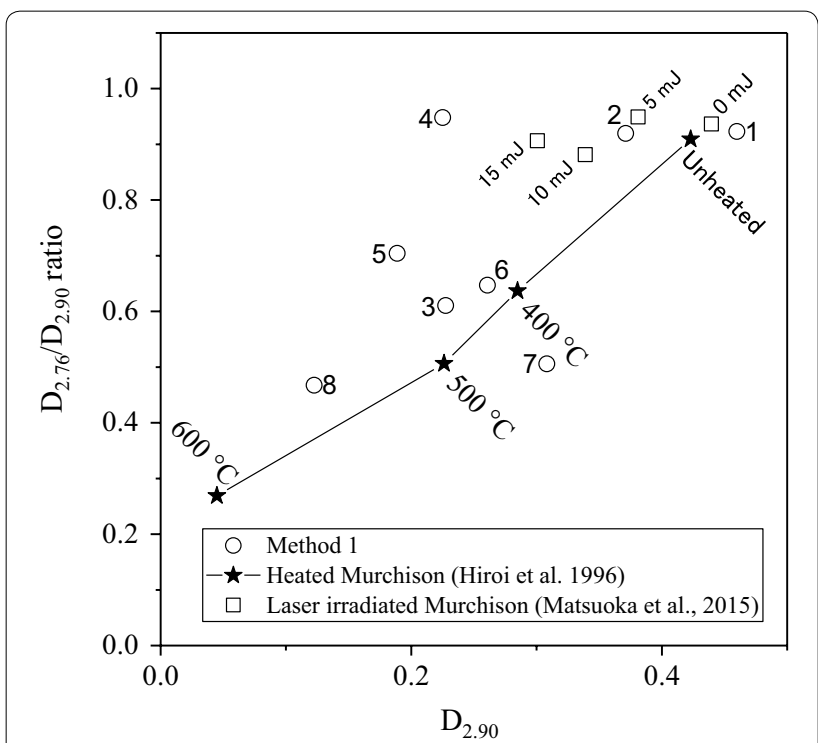

Fig. $12 D_{2.90}$ values and the $D_{2.76} / D_{2.90}$ ratios of NIRS3 data of the meteorite samples corrected by the method 1, plotted with those of FT-IR spectra of Murchison samples heated in vacuum (up to $600^{\circ} \mathrm{C}$, Hiroi et al. 1996) and irradiated by pulse laser (Matsuoka et al. 2015). Labels: $1=$ Murchison, $2=$ Murray, $3=Y-793321,4=J$ bilet Winselwan, $5=Y 982086,6=Y 980115,7=Y-86720$, and $8=B-7904$ clue for understanding the surface properties of Ryugu and the relationship between $\mathrm{C}$-type asteroids and $\mathrm{CM}$ chondrites.

\section{Abbreviations}

NIRS3: Near-Infrared Spectrometer; FT-IR: Fourier transform infrared; ONC-T: Optical Navigation Camera Telescope; TIR: Thermal Infrared Imager; UV: ultraviolet; Vis: visible; NIR: near-infrared; IRTF: Infrared Telescope Facility; (NIRS3-) $\mathrm{S}$ : the spectrometric unit; (NIRS3-) AE: the analog electric unit; (NIRS3-) HNS: a harness cables; InAs: indium arsenide; MGM: modified Gaussian model.

\section{Authors' contributions}

$\mathrm{MM}, \mathrm{TN}$, and TO contributed to sample preparation, data collection, analyses, and correction, and manuscript preparation. TI, KK, and MA contributed to the data collection and analyses. YN and TA contributed to the data collection, analyses, and correction. MK participated in data collection. TH contributed to the data collection and analyses. IN, AY, and HK contributed to sample preparation. All authors read and approved the final manuscript.

\section{Author details}

${ }^{1}$ Tohoku University, Sendai, Miyagi 980-8578, Japan. ${ }^{2}$ Japan Atomic Energy Agency, Tokai, Ibaraki 319-1195, Japan. ${ }^{3}$ Japan Aerospace Exploration Agency, Sagamihara, Kanagawa 252-5210, Japan. ${ }^{4}$ The University of Aizu, Aizu-Wakamatsu, Fukushima 965-8580, Japan. ${ }^{5}$ National Institute for Environmental Studies, Tsukuba, Ibaraki 305-8506, Japan. ${ }^{6}$ Graduate University for Advanced Studies, Hayama, Kanagawa 240-0193, Japan. ${ }^{7}$ Brown University, Providence, RI 02912, USA. ${ }^{8}$ National Institute of Polar Research, Tachikawa, Tokyo 190-8518, Japan. ${ }^{9}$ Department of Polar Science, School of Multidisciplinary Science, SOKENDAI (The Graduate University for Advanced Studies), Tokyo 190-8518, Japan.

\section{Acknowledgements}

We wish to thank at Meisei Co., Ltd., especially to Dr. Hajime Murao, Ms. Yuko Minagawa-Sakata, and Mr. Koji Taguchi.

\section{Competing interests}

The authors declare that they have no competing interests.

Received: 31 May 2017 Accepted: 18 August 2017

Published online: 04 September 2017

\section{References}

Burbine T, McCoy T, Meibom A, Gladman B, Keil K (2002) Meteoritic parent bodies: their number and identification. Asteroids III. University of Arizona Press, Tucson, pp 653-667

Bus SJ, Binzel RP (2002) Phase II of the small main-belt asteroid spectroscopic survey: a feature-based taxonomy. Icarus 158(1):146-177

Clark RN, King TVV, Klejwa M, Swayze GA, Vergo N (1990) High spectral resolution reflectance spectroscopy of minerals. J Geophys Res 95(B8):12653-12680

Hiroi T, Pieters CM, Zolensky ME, Lipschutz ME (1993) Evidence of thermal metamorphism on the $\mathrm{C}, \mathrm{G}, \mathrm{B}$, and $\mathrm{F}$ asteroids. Science 261(5124):1016-1018

Hiroi T, Pieters CM, Zolensky ME, Lipschutz ME (1996) Thermal metamorphism of the $C, G, B$, and $F$ asteroids seen from the $0.7-\mu m, 3-\mu m$, and UV absorption strengths in comparison with carbonaceous chondrites. Meteorit Planet Sci 31:321-327

Iwata T, Kitazato K, Abe M, Ohtake M, Arai T, Arai T, Hirata N, Hiroi T, Honda C, Imae N, Komatsu M, Matsunaga T, Matsuoka M, Matsuura S, Nakamura T, Nakato A, Nakauchi Y, Osawa T, Senshu H, Takagi Y, Tsumura K, Takato N, Watanabe S, Barucci M, Palomba E, Ozaki M (2017) 
NIRS3: the near infrared spectrometer on Hayabusa2. Space Sci Rev 208:317-337

Jones TD, Lebofsky LA, Lewis JS, Marley MS (1990) The composition and origin of the $C, P$, and $D$ asteroids: water as a tracer of thermal evolution in the outer belt. Icarus 88(1):172-192

Kitazato K, Iwata T, Abe M, Ohtake M, Matsuura S, Arai T, Nakauchi Y, Tsumura K, Hirata N, Senshu H, Watanabe S, Hayabusa-2 NIRS3 Team (2015) In-flight performance of the Hayabusa-2 near-infrared spectrometer (NIRS3). In: Abstracts of the 46th lunar planetary science conference, The Woodlands, 16-20 March 2015

Lantz C, Clark BE, Barucci MA, Lauretta DS (2013) Evidence for the effects of space weathering spectral signatures on low albedo asteroids. Astron Astrophys 554:1-7

Lazzaro D, Barucci MA, Perna D, Jasmim FL, Yoshikawa M, Carvano JMF (2013) Rotational spectra of (162173) $1999 \mathrm{JU} 3$, the target of the Hayabusa2 mission. Astron Astrophys 549:L2

Matsuoka M, Nakamura T, Kimura Y, Hiroi T, Nakamura R, Okumura S, Sasaki S (2015) Pulse-laser irradiation experiments of Murchison CM2 chondrite for reproducing space weathering on C-type asteroids. Icarus 254:135-143

Matsuoka M, Nakamura T, Miyajima N, Imae N, Yamaguchi A, Kojima H (2017) Vis-IR reflectance spectroscopy of hydrous carbonaceous chondrites with variable heating and dehydration degrees. In: Abstracts of the 48th lunar and planetary science conference, The Woodlands, 20-24 March 2017

Miyamoto M (1992) Infrared diffuse reflectance spectra of several thermally metamorphosed carbonaceous chondrites. Antarct Meteor Res 5:155-164

Miyamoto M, Zolensky ME (1994) Infrared diffuse reflectance spectra of carbonaceous chondrites: amount of hydrous minerals. Meteoritics 29(6):849-853

Nakamura T (2005) Post-hydration thermal metamorphism of carbonaceous chondrites. J Miner Petrol Sci 100(6):260-272

Nakamura T, Iwata T, Kitazato K, Abe M, Osawa T, Matsuoka M, Nakauchi Y, Arai T, Komatsu M, Hiroi T, Imae N (2015) Reflectance spectra measurement of various carbonaceous chondrites using Hayabusa-2 near infrared spectrometer. In: Abstracts of the 78th annual meeting of the meteoritical society, Berkeley, 27-31 July 2015

Okamura N, Sugita S, Kamata S, Usui F, Hiroi T, Ootsubo T, Müller TG, Sakon I, Hasegawa S (2014) Principal-component analysis of the continuous 3- $\mu \mathrm{m}$ spectra of low-albedo asteroids observed with the AKARI satellite. In: Abstracts of the 45th lunar planetary science conference, The Woodlands, 17-21 March 2014

Osawa T, Kagi H, Nakamura T, Noguchi T (2005) Infrared spectroscopic taxonomy for carbonaceous chondrites from speciation of hydrous components. Meteorit Planet Sci 40(1):71-86
Perna D, Barucci MA, Ishiguro M, Alvarez-Candal A, Kuroda D, Yoshikawa M, Kim M-J, Fornasier S, Hasegawa S, Roh D-G, Müller TG, Kim Y (2017) Spectral and rotational properties of near-Earth asteroid (162173) Ryugu, target of the Hayabusa2 sample return mission. Astron Astrophys 599:L1

Rivkin AS, Davies JK, Johnson JR, Ellison SL, Trilling DE, Brown RH, Lebofsky LA (2003) Hydrogen concentrations on C-class asteroids derived from remote sensing. Meteorit Planet Sci 38(9):1383-1398

Rivkin AS, Volquardsen EL, Clark BE (2006) The surface composition of Ceres: discovery of carbonates and iron-rich clays. Icarus 185(2):563-567

Sugita S, Kuroda D, Kameda S, Hasegawa S, Kamata S, Abe M, Ishiguro M, Takato N, Yoshikawa M (2013) Visible spectroscopic observation of asteroid 162173 (1999JU3) with the Gemini-s telescope. In: Abstracts of the 44th lunar planetary science conference, The Woodlands, 18-22 March 2013

Sunshine JM, Pieters CM, Pratt SF (1990) Deconvolution of mineral absorption bands: an improved approach. J Geophys Res 95:6955-6966

Takir D, Emery JP (2012) Outer main belt asteroids: identification and distribution of four 3- - m spectral groups. Icarus 219(2):641-654

Takir D, Emery JP, McSween HY, Hibbitts CA, Clark RN, Pearson N, Wang A (2013) Nature and degree of aqueous alteration in CM and Cl carbonaceous chondrites. Meteorit Planet Sci 48(9):1618-1637

Tsuda Y, Yoshikawa M, Abe M, Minamino H, Nakazawa S (2013) System design of the Hayabusa 2-asteroid sample return mission to $1999 \mathrm{JU} 3$. Acta Astronaut 91:356-362

Vilas F (2008) Spectral characteristics of Hayabusa 2 near-earth asteroid targets 1621731999 JU3 and 2001 QC34. Astron J 135(4):1101-1105

Vilas F, Gaffey MJ (1989) Phyllosilicate absorption features in main-belt and outer-belt asteroid reflectance spectra. Science 246:790-792

Vilas F, Jarvis KS, Gaffey MJ (1994) Iron alteration minerals in the visible and near-infrared spectra of low-albedo asteroids. Icarus 109(2):274-283

Yamashita S, Nakamura T, Jogo K, Matsuoka M, Okumura S (2015) Progressive changes in mineralogy, reflectance spectra and water contents of experimentally heated murchison at 400,600 , and $900^{\circ} \mathrm{C}$. In: Abstracts of 78th annual meeting of the meteoritical society, Berkeley, 27-31 July 2015

Zolensky M, Barrett R, Browning L (1993) Mineralogy and composition of matrix and chondrule rims in carbonaceous chondrites. Geochim Cosmochim Acta 57:3123-3148

\section{Submit your manuscript to a SpringerOpen ${ }^{\circ}$ journal and benefit from:}

- Convenient online submission

- Rigorous peer review

- Open access: articles freely available online

- High visibility within the field

- Retaining the copyright to your article

Submit your next manuscript at $\boldsymbol{\nabla}$ springeropen.com 